\title{
Nursing Challenges in Interactions with Patients Receiving Mechanical Circulatory and Respiratory Support
}

\author{
Cara Wrigley, PhD; Karla Straker, PhD; Erez Nusem, PhD; John F. Fraser, MD, PhD; \\ Shaun D. Gregory, PhD
}

\begin{abstract}
Background: Mechanical circulatory and respiratory support (MCRS) systems provide short- to long-term life support for patients with severe cardiac or respiratory failure. Whereas the challenges of patients with these lifesaving systems are well understood, the challenges faced by nurses of patients receiving MCRS remain relatively unidentified. Objectives: In this study, we investigated the challenges and experiences of MCRS nurses, with the aim of informing the design of future interventions. Design: A qualitative approach was taken to develop an in-depth understanding of the emotional issues nurses experience in their daily interactions with patients. Participants and Setting: Nine MCRS nurses ranging in clinical experience were recruited from Australian hospitals. Methods: Participants were divided into 3 focus groups, with design prompts being used in the focus groups to facilitate discussion. Data from these focus groups were analyzed through a thematic analysis protocol. Results: The nurses' accounts were clustered around 2 themes, including (1) patient connection and (2) compassion vitality and fatigue. Each theme elicits a conflicting compromise that MCRS nurses face daily. Conclusion: The challenges of nurses who support patients with MCRS are complex, multifaceted, emotionally stimulating, and exhausting. We therefore contributes a set of design criteria to support such nurses, setting a direction for future research.

KEY WORDS: extracorporeal membrane oxygenation, mechanical circulatory and respiratory support, nursing, ventricular assist devices
\end{abstract}

W ithin the domain of mechanical circulatory and respiratory support (MCRS), ventricular assist devices (VADs) are increasingly being used to sustain circulatory function in patients waiting for a cardiac transplant either as a destination therapy or as a bridge

Cara Wrigley, PhD

Sydney School of Architecture, Design \& Planning, The University of Sydney, Australia.

Karla Straker, PhD

Sydney School of Architecture, Design \& Planning, The University of Sydney, Australia.

Erez Nusem, PhD

Sydney School of Architecture, Design \& Planning, The University of Sydney, Australia.

John F. Fraser, MD, PhD

Innovative Cardiovascular Engineering and Technology Laboratory, Critical Care Research Group, The Prince Charles Hospital, Brisbane; and School of Medicine, The University of Queensland, Australia.

Shaun D. Gregory, PhD

Innovative Cardiovascular Engineering and Technology Laboratory, Critical Care Research Group, The Prince Charles Hospital, Brisbane; School of Medicine, The University of Queensland; and School of Engineering, Griffith University, Southport, Australia.

The authors have no funding or conflicts of interest to disclose. Correspondence

Cara Wrigley, PhD, Sydney School of Architecture, Design and Planning, University of Sydney, Wilkinson Building (G04), 148 City Road, NSW 2006 (cara.wrigley@sydney.edu.au).

DOI: $10.1097 / J C N .0000000000000494$ to recovery or decision. ${ }^{1}$ Similarly, extracorporeal membrane oxygenation (ECMO) provides short-term support for the lungs with/without circulatory support as a bridge to recovery, decision, or transplant. Although different technologies, patients with both VAD and ECMO face similar issues, requiring intensive physical care and a great deal of nursing and psychological support. ${ }^{2}$ Subsequently, nurses of both VAD and ECMO patients are subject to similar challenges.

Complications among patients receiving MCRS are common, with patients requiring constant monitoring. It is not uncommon for patients to be critically ill for extensive periods and to require considerable attention in intensive care units. Nursing such patients is a complex, multifaceted, and intense occupation. Nurses employed in this field are intimately involved in the MCRS process, providing support not only to patients but also to caregivers and family members. The nurse's role in the end-of life decision-making process can be ethically challenging and emotionally demanding. ${ }^{3}$ However, despite the increasing popularity of MCRS technologies, a growing number of patients (eg, yearly VAD implants in the United States have risen from $<100$ in 2006 to $>2500$ in 2013), and improved patient outcomes, ${ }^{4}$ investigators have yet to address how these nurses deal with a constant contention between their love for the job and the 
emotional strain it places upon them. We therefore aim to explore the spectrum of MCRS nurses' experiences (ie, their interactions with both inpatients and outpatients, at all stages of MCRS).

\section{Related Literature}

Mechanical circulatory and respiratory support systems can have multifaceted issues, including device/circuit failures, bleeding, infection, stroke, death, thrombosis, and quality-of-life issues. ${ }^{5}$ Such high complication rates with MCRS result in an intensive treatment regime, where those who manage the patients are exposed to unique workplace challenges. Despite this myriad of challenges, little information was found pertaining to a nurse's challenges with MCRS. Some studies have, however, explored the experiences of carers and family members of VAD patients. ${ }^{6,7}$

A study conducted by Gibson et $\mathrm{al}^{8}$ revealed 4 key topics that highlight the unique issues faced by nurses of VAD patients: exclusive knowledge, human condition, ethics, and interdisciplinary stress. The study concluded that institutions with VAD programs should consider providing emotional support for nurses associated with VAD patients because the work is often emotionally challenging. Prominent among these topics were (i) exclusive knowledge and (ii) human condition. Of these 2 topics, exclusive knowledge outlined the unique knowledge VAD nurses develop through direct exposure to patients and the medical team. This knowledge relates to technical issues of a VAD and also a "special sense of knowing" about a patient's foreseeable experience. The latter topic, human condition, is characterized by the issues of patient relationships and compassion fatigue. The issue of patient relationships explores intense and prolonged nurse-patient relationships, which often result in heightened distress and strain on VAD nurses. Compassion fatigue relates to the emotional reactions that can result from direct and prolonged exposure to patients who are very ill and experience adverse events. The significance of witnessing a patient suffering is explored in this article to understand how nurses cope with "typical" and "bad" experiences. ${ }^{8}$

One such coping mechanism is a nurse's personal resilience (ie, "a person's determination and ability to endure, to be adaptable and to recover from adversity"), ${ }^{9(\mathrm{p} 347)}$ a concept well detailed in literature, with numerous prominent frameworks to assist nurses in developing resiliency. ${ }^{9-11}$ However, MCRS nurses are generally subject to a greater load and spectrum of strain than that of the average nurse, requiring a greater deal of support. Questions remain surrounding the challenges and burdens placed on nurses of patients receiving MCRS. This study was therefore structured to empathize with MCRS nurses to better understand their experiences and challenges and synthesize these into a set of design criteria to inform future work.

\section{Methodology}

\section{Aims and Objectives}

The methodological basis for this study was interpretive phenomenological analysis. Interpretive phenomenological analysis is an approach to qualitative analysis that focuses on how people make sense of their experiences, offering an established, phenomenologically focused method for interpretation. ${ }^{8,12}$ Focus groups were conducted with 9 MCRS nurses, satisfying interpretive phenomenological analysis requirement of reflective first-person accounts from participants. These focus groups had the objective of providing an in-depth qualitative perspective on the current challenges nurses face when caring for MCRS patients. We aimed to:

- explore the main issues of MCRS nurses in Australia across 3 topics synthesized from literature (ie, exclusive knowledge, patient relationships, and compassion fatigue); and

- identify design criteria with the objective of informing future work and assisting MCRS nurses in their daily interactions with patients.

\section{Protocol}

Three focus groups were formed to explore each of the 3 aforementioned topics. The focus group consisted of 3 nurses, with a different author facilitating each focus group and 1 author floating between the focus groups to ensure the protocol was followed. The focus groups were structured around a set of prompts (quotes and findings from the literature review). Participants were recruited through an existing relationship with the researchers, with each participant being allocated into a focus group based on their area of expertise, and asked to discuss their experiences and perspectives. The focus groups were completed over a 2-hour period and had minimal intervention from the facilitator. Each focus group was recorded and transcribed for analysis.

\section{Analysis}

Analysis of the data followed an interpretive phenomenological analysis protocol. ${ }^{12}$ Focus group transcripts were individually coded by three of the authors, with a primary focus on coding experiential content. An initial set of patterns within each of the transcripts was identified, leading to the formation of first-order codes. These codes were then synthesized and collapsed into more abstract themes. Cumulative patterns across the themes in each of the transcripts were identified, and a coding structure was formed. Investigator triangulation was achieved by crossanalyzing data from focus groups. ${ }^{13}$ 


\section{Participants}

As MCRS nurses require highly specialized training, participants from 5 Australian hospitals (The Prince Charles Hospital, Brisbane; St Vincent's Hospital, Sydney; The Royal Melbourne Children's Hospital; The Alfred Hospital, Melbourne; and The Fiona Stanley Hospital, Perth) were recruited for this study (see Table 1). To be eligible for inclusion in the study, each participant was required to have practiced as a specialized VAD or ECMO nurse. The study was approved by the University of Sydney's Ethics Office (reference number 2017/733) and received the consent of each of the participating nurses. We present a multicenter study, involving participants with a wide range of expertise. Most participating nurses had more than 10 years of professional nursing experience.

\section{Findings}

The findings are presented as themes and quotations elucidated from focus group participants. This article reports on 2 prominent themes that emerged from the research: (1) patient connection and (2) compassion vitality and fatigue.

\section{Patient Connection}

The theme of patient connection explores the dichotomy between a nurse's need to form a personal connection with his/her patient receiving MCRS and his/her ability to disconnect from these patients in personal downtime. Achieving a balance between these 2 dimensions is critical in delivering a professional level of care.

Nurses reported that a personal connection with a patient receiving MCRS intrinsically linked to their job, because their duties sometimes fall outside the regular scope of nursing. The needs of such patients are often complex and challenging to meet. Indeed, in relation to meeting these needs, 1 nurse stated that "you do what you can for them. Sometime it is out of the realms of nursing and sometimes it isn't - that's again why you do seem to find a bond with them." With the prognosis of patients often being uncertain, nurses feel that it is their duty to form a meaningful bond and to "do the best to improve their life while they're waitingbecause they could have a stroke the next day and die." Because some of these relationships continue until the patient's death, the outlook detailed can be quite daunting. Despite this prospective issue, not rushing and taking time to be present with patients is a critical aspect of providing quality care.

Building empathy can also help to deliver more tailored care. Indeed, as explained by 1 participant, "I empathise as to what they must be going through, what they must be feeling, therefore that makes me think a little bit more outside the box on what I can do for them." This role also extends to the patient's family, where the nurse assumes the role of a translator of medical information and provides emotional support. In some cases, such a role blurs the line between friend and professional and has resulted in nurses not receiving the courtesy given to other medical professionals. One nurse exclaimed that "they're not necessarily on their best behaviour with us because they feel like we're almost closer. You know how you take your family for granted, even though you love them? It's that sort of relationship." These relationships are challenging to manage and are a unique aspect of nursing associated with caring for patients with MCRS.

Interactions with patients are long-term, with many nurses explaining that they have become part of their patients' lives. These connections form as patients receiving MCRS and nurses share their worlds with one another. Forming connections with patients is integral in providing support and comfort, and although these relationships can be rewarding, they also hold an intrinsic burden. Knowing so much about patients and their lives and being there as they pass away or face adversity strain nurses. Nurses are constantly mindful of this potential outcome because the survival rate of patients receiving MCRS is comparatively low with other therapies, and

\begin{tabular}{|c|c|c|c|c|c|}
\hline \multicolumn{6}{|c|}{ Participants } \\
\hline $\begin{array}{l}\text { Focus } \\
\text { Group }\end{array}$ & Nurse No. & Current Role & Time in Position & PNE, y & Previous Roles \\
\hline \multirow[t]{3}{*}{ A } & 1 & Clinical nurse ECMO CNC & 10 y 5 mo & 19 & RN ICU EN CSSD \\
\hline & 2 & MCS ICU & $13 y$ & 11 & CNS RN \\
\hline & 3 & VAD nurse researcher & $1 \mathrm{y}$ & 19 & Research coordinator RN \\
\hline \multirow[t]{3}{*}{ B } & 4 & ECMO CNC & $15 y$ & 19 & Nurse educator CNS \\
\hline & 5 & VAD coordinator & $12 \mathrm{y}$ & 11 & ICU nurse Nurse educator Clinical nurse \\
\hline & 6 & MCS nurse & $12 \mathrm{y}$ & 13 & Clinical nurse ICU Clinical nurse CCU \\
\hline \multirow[t]{3}{*}{ C } & 7 & Nurse MCS & $10 \mathrm{y}$ & 11 & CNC VAD transplant CNS coronary care \\
\hline & 8 & ECMO clinical nurse & $7 y$ & 16 & Clinical ICU educator Critical care nurse \\
\hline & 9 & MCS nurse & $3 y$ & None & ICU nurse \\
\hline
\end{tabular}

Abbreviations: CCU, critical care nurse; CNC, clinical nurse consultant; CNS, clinical nurse specialist; CSSD, Central Sterile Supplies Department; ECMO, extracorporeal membrane oxygenation; EN, enrolled nurse; ICU, intensive care unit; PNE, professional nursing experience; RN, registered nurse; VAD, ventricular assist device. 
indeed, as said by a participating nurse: "That is something for me to remind myself because as you say, if something goes tragically wrong for these patients, you put yourself up for an awful lot of heartache." Understanding and balancing these relationships was noted as a challenge, with several nurses explaining that "it's very hard to take a step back" and "knowing when to stop is not always easy." Providing quality care and maintaining professionalism in the face of such issues were linked to experience: "I think probably from our experience in the past we would probably be able to identify some pitfalls in developing relationships and what to look for and what are warning signs." However, even experience did not guarantee a balance because "backing off emotionally is sometimes hard, particularly if you actually really like them." Although not every experience with a patient is positive, a nurse-patient relationship can be incredibly rewarding and is essential in delivering a high standard of care.

\section{Compassion Vitality and Fatigue}

Mechanical circulatory and respiratory support nurses play a critical role in the continuation of life of their patients, yet performing this role can be emotionally exhausting. Hence, the emotional vitality of these nurses is challenged, which often results in compassion fatigue.

One source of such fatigue was noted as an influx of patients, leading to the nurse giving "optimal care whilst having compassion fatigue." Behaviors noted as "compassion towards your patient and you being unable to deliver that to your patient because you are so emotionally stressed out." A particular challenge of compassion was stated as having "to be able to divorce yourself and be professional, but be compassionate and caring, but then park it and go home and not take that home to your own family." The toll it then takes on the nurse's personal life stems from being unable to communicate and share this burden: "Your personal relationships suffer because you take it home."

Several nurses found that their relationships, both collegial and personal, suffered as a result of compassion fatigue, which "impacts your ability to perform your job because your confidence is knocked or maybe you don't want to put yourself in that situation again so you draw back." Even with nurses in long-term relationships, many partners could not comprehend the obligation felt by these nurses, with a nurse stating: "He does not get that you can't leave work and be done. We have been together 30 years now and even then, he will say really [Nurse's Name]?" The obligation stems first from patients and then secondarily from fellow nurses on roster with comments along the line of "if I don't take this call or tell them where to find particular equipment that could impact on a patient's outcome." Trying to justify and explain this to family and friends was found to be difficult because they did not understand.

"Burnout" of MCRS nurses was documented as not only "being common" but also as "losing your love of why you were there in the first place." It was said to occur "when you have pretty full on patient scenarios, day after day" and "not only full on patients but also full on families; dealing with the emotional side of ECMO or VAD in someone acutely ill with families... who don't get along or who don't understand what's happening." Despite management attempting to prevent such occurrences and effect in these nurses, it was said to still exist- "as hard as management try to minimise it still definitely does happen.”

Mechanical circulatory and respiratory support nurses require special training and are perceived as incredibly capable due to the demanding nature of these particular patients: "If you can handle a busy ECMO patient you are a good nurse." With such a specialized skill set, MCRS nurses are sometimes singled out and made to feel obligated to come into work outside their rostered periods - "Anyone who is ECMO trained please put your hand up to come in... you feel like there is only so many of us." This results in "always checking your phone and emails even when you have days off...you never really turn off." Despite special training, these nurses feel that they require support in dealing with their emotional burdens. In addition, as patient advocates, these nurses feel emotionally obligated to be "the voice of the patient," even when this entails taking a contradictory argument to that of the surgeon or cardiologist, "so you feel like you are conflicted between the patient and the medical staff and the family."

Mechanical circulatory and respiratory support nurses generally discuss their issues with others in their field, as their perception is that only MCRS nurses understand each other and their challenges. Currently, these issues are resolved in regular cohort support groups of nurses, where they debrief over tea or wine. As articulated by a participating nurse, "It's informal, but it's from people who understand."

\section{Implications and Recommendations}

Our findings were split across the themes of (a) patient connection and (b) compassion vitality and fatigue, with these themes exploring $(a)$ the dichotomy between a nurse's need to form a relationship with a patient and the long-term impact of such a relationship and $(b)$ the emotional connotations of MCRS nurse-patient relationships. Through these themes, we illustrate the "doubleedged" and paradoxical nature of problems MCRS nurses face- a perspective yet to be explored in literature. The findings indicate that, to deliver the highest quality of care and maintain professionalism, MCRS nurses must form 
bonds with their patients. However, it can be challenging to disconnect from these bonds, which can inevitably become an emotional and professional burden with implications that extend beyond a nurse's professional life.

Although nurses' personal resilience (the facet of recuperability in particular ${ }^{14}$ ) plays a large role in assisting to cope with the challenges patients receiving MCRS, it is insufficient as a standalone mechanism. Subsequently, there is a significant opportunity for future work to assist these nurses in managing the issues of patient connection, and compassion vitality and fatigue.

Derived from the findings are recommendations made by the MCRS nurses and researchers in overcoming some of the issues described in the findings. Table 2 outlines these challenges and their impact, along with a set of recommendations to achieve the overall aims for each theme. These recommendations have been developed into 4 design criteria, which address the unique contextual problems of MCRS nurses; these include (1) the discovery and automation of maintaining awareness of nurses' emotional state, (2) communicating information to the right people at appropriate times, (3) enabling directed help or intervention when required, and (4) integration into daily routine.

The design criteria developed through this research will inform future work, including the design of a channel for respite and emotional expression and the development of an official national and international communication platform between MCRS nurses as a means of sharing knowledge and support provision.

\section{Conclusions}

This article explored the themes of patient connection, and compassion vitality and fatigue of MCRS nurses in their daily interactions with patients. The challenges outlined in these themes host a cascading effect to the optimal care given by these nurses - which takes a toll on nurses in the form of emotional burnout and impacts the quality of care received by patients. The design

\section{TABLE 2 Synthesis of Mechanical Circulatory and Respiratory Support Nurse Challenges and Recommendations}

\begin{tabular}{|c|c|c|c|c|}
\hline Theme & Challenge & Impact & Recommendation & $\begin{array}{l}\text { Design } \\
\text { Criteria }\end{array}$ \\
\hline \multirow{4}{*}{$\begin{array}{l}\text { Patient connection } \\
\text { Aim: to assist nurses in } \\
\text { achieving a balance } \\
\text { between forming a } \\
\text { personal connection with } \\
\text { their MCRS patient and } \\
\text { their ability to disconnect } \\
\text { from these patients in } \\
\text { personal downtime }\end{array}$} & $\begin{array}{l}\text { Not knowing when to } \\
\text { take a step back and } \\
\text { disconnect }\end{array}$ & $\begin{array}{l}\text { Nurses overinvested in their } \\
\text { patients, detrimental to } \\
\text { their well-being when } \\
\text { patients face adversity }\end{array}$ & $\begin{array}{c}\text { The discovery and automation } \\
\text { of maintaining awareness } \\
\text { of nurses' emotional state }\end{array}$ & 1 \\
\hline & $\begin{array}{l}\text { Blurred line between } \\
\text { friend and professional }\end{array}$ & $\begin{array}{l}\text { Nurses not receiving the } \\
\text { courtesy given to other } \\
\text { medical professionals }\end{array}$ & $\begin{array}{l}\text { Delivery time, tone, and } \\
\text { content of communication } \\
\text { appropriate for context }\end{array}$ & 2 \\
\hline & $\begin{array}{l}\text { Constantly mindful of } \\
\text { survival rates of MCRS } \\
\text { patients }\end{array}$ & $\begin{array}{l}\text { Lower empathy for patients } \\
\text { to protect emotional } \\
\text { well-being }\end{array}$ & $\begin{array}{l}\text { Changing the time frames } \\
\text { between interactions to } \\
\text { allow for recovery }\end{array}$ & 3 \\
\hline & $\begin{array}{l}\text { Nurses feel that it is their } \\
\text { duty to form a } \\
\text { meaningful bond with } \\
\text { their patients }\end{array}$ & $\begin{array}{l}\text { Nurses required to provide } \\
\text { support outside the scope } \\
\text { of their professional duties } \\
\text { but deliver more tailored } \\
\text { and innovative care }\end{array}$ & $\begin{array}{l}\text { Integration of supplementary } \\
\text { duties into daily routine to } \\
\text { assist in building empathy } \\
\text { with patient }\end{array}$ & 4 \\
\hline \multirow[t]{4}{*}{$\begin{array}{l}\text { Compassion vitality and fatigue } \\
\text { Aim: improving the } \\
\text { emotional vitality of } \\
\text { nurses to decrease } \\
\text { compassion fatigue }\end{array}$} & $\begin{array}{l}\text { Obligated to be "the voice } \\
\text { of the patient," even } \\
\text { when this entails } \\
\text { contradicting the } \\
\text { surgeon or cardiologist }\end{array}$ & $\begin{array}{l}\text { Strained professional relationships, } \\
\text { conflicted between the } \\
\text { patient, medical staff, } \\
\text { and family }\end{array}$ & $\begin{array}{l}\text { Having a designated channel } \\
\text { for the patient wishes to } \\
\text { be respected }\end{array}$ & 1 \\
\hline & $\begin{array}{l}\text { Partners and family } \\
\text { members unable to } \\
\text { empathize and } \\
\text { comprehend the nurses' } \\
\text { sense of obligation }\end{array}$ & $\begin{array}{l}\text { Difficulty in disconnecting } \\
\text { impacts personal life and } \\
\text { results in personal } \\
\text { relationships suffering }\end{array}$ & $\begin{array}{l}\text { Communicating and sharing } \\
\text { this burden and associated } \\
\text { challenges with professionals } \\
\text { who can relate }\end{array}$ & 2 \\
\hline & $\begin{array}{l}\text { Influx of patients, and difficult } \\
\text { patient scenarios and } \\
\text { family interactions }\end{array}$ & $\begin{array}{l}\text { Having to deal with the } \\
\text { emotional, operational, and } \\
\text { personal relations while also } \\
\text { providing medical care }\end{array}$ & $\begin{array}{l}\text { Integration of an outsider } \\
\text { (ie, specialized counselor/ } \\
\text { support network) to } \\
\text { moderate relationships } \\
\text { with patients' families }\end{array}$ & 3 \\
\hline & $\begin{array}{l}\text { Patient outcomes drive } \\
\text { obligation to work outside } \\
\text { roster; nurses endeavor } \\
\text { to always be contactable }\end{array}$ & $\begin{array}{l}\text { Never really "turning off" } \\
\text { resulting in burnout }\end{array}$ & $\begin{array}{l}\text { Providing consistent support } \\
\text { to allow nurses to mentally } \\
\text { disconnect }\end{array}$ & 4 \\
\hline
\end{tabular}




\section{What's New and Important}

Patient connection, and compassion vitality and fatigue contain a conflicting compromise that MCRS nurses face daily - the gift of resilience.

- The challenges of nurses who support patients with MCRS are complex, multifaceted, emotionally stimulating, and exhausting.

- This article contributes a set of design recommendations to support such nurses, setting a direction for future research.

criteria listed here should be explored further to inform a future solution that addresses the complex challenges faced by MCRS nurses.

\section{Acknowledgments}

The authors thank the MCRS nurses from around Australia who took part in this study. The authors would like to recognize the financial assistance provided by The Prince Charles Hospital Foundation (PRO2014-08) and the National Health and Medical Research Council Centre for Research Excellence (APP1079421).

\section{REFERENCES}

1. Barnes K. Complications in patients with ventricular assist devices. Dimens Crit Care Nurs. 2008;27(6):233-241.

2. Chapman E, Parameshwar J, Jenkins D, Large S, Tsui S. Psychosocial issues for patients with ventricular assist devices: a qualitative pilot study. Am J Crit Care. 2007;16(1):72-81.

3. Bisal M. Nurses with heart. Nurs BC/Registered Nurses Assoc Br Columbia. 2008;40(4):14-20.
4. Kirklin JK, Naftel DC, Pagani FD, et al. Seventh INTERMACS annual report: 15,000 patients and counting. J Hear Lung Transplant. 2015;34(12):1495-1504.

5. Kirklin JK, Pagani FD, Kormos RL, et al. Eighth annual INTERMACS report: special focus on framing the impact of adverse events. J Hear Lung Transplant. 2017;36(10): 1080-1086.

6. Bunzel B, Laederach-Hofmann K, Wieselthaler GM, Roethy W, Drees G. Posttraumatic stress disorder after implantation of a mechanical assist device followed by heart transplantation: evaluation of patients and partners. Transplant Proc. 2005;37(2):1365-1368.

7. Casida J. The lived experience of spouses of patients with a left ventricular assist device before heart transplantation. Am J Crit Care. 2005;14(2):145-152.

8. Gibson JA, Henderson A, Jillings C, Kaan A. Nursing patients with ventricular assist devices: an interpretive description. Prog Transplant. 2013;23(2):147-153.

9. Dyess SM, Prestia AS, Smith MC. Support for caring and resiliency among successful nurse leaders. Nurs Adm Q. 2015; 39(2):104-116.

10. Stagman-Tyrer D. Resiliency and the nurse leader: the importance of equanimity, optimism, and perseverance. Nurs Manage. 2014;45(6):46-50.

11. Turner SB, Kaylor SD. Neuman systems model as a conceptual framework for nurse resilience. Nurs Sci Q. 2015; 28(3):213-217.

12. Larkin $\mathrm{M}$, Thompson A. Interpretative phenomenological analysis in mental health and psychotherapy research. In: Thompson A, Harper D, eds. Qualitative Research Methods in Mental Health and Psychotherapy: A Guide for Students and Practitioners. Oxford, UK: John Wiley \& Sons; 2012:99-116.

13. Thurmond VA. The point of triangulation. J Nurs Scholarsh. 2001;33(3):253-258.

14. Wei W, Taormina RJ. A new multidimensional measure of personal resilience and its use: Chinese nurse resilience, organizational socialization and career success. Nurs Inq. 2014;21(4):346-357. 